\title{
Digital signal processing based on inverse scattering transform
}

\author{
Elena G. Turitsyna* and Sergei K. Turitsyn \\ Aston Institute of Photonic Technologies, Aston University, Birmingham B4 7ET, UK \\ ${ }^{*}$ Corresponding author: e.g.turitsyna@aston.ac.uk
}

Received July 4, 2013; revised August 30, 2013; accepted September 16, 2013; posted September 17, 2013 (Doc. ID 193344); published October 11, 2013

\begin{abstract}
Through numerical modeling, we illustrate the possibility of a new approach to digital signal processing in coherent optical communications based on the application of the so-called inverse scattering transform. Considering without loss of generality a fiber link with normal dispersion and quadrature phase shift keying signal modulation, we demonstrate how an initial information pattern can be recovered (without direct backward propagation) through the calculation of nonlinear spectral data of the received optical signal. (c) 2013 Optical Society of America

OCIS codes: (060.2330) Fiber optics communications; (060.1660) Coherent communications; (070.4340) Nonlinear optical signal processing.

http://dx.doi.org/10.1364/OL.38.004186
\end{abstract}

The technology of coherent optical communications has enabled a dramatic improvement of fiber-optic transmission systems. The introduction of advanced modulation formats [1] and digital signal processing (DSP) for coherent communications (see, e.g., [2-4 ] and references therein) led to practical implementation of systems with $100 \mathrm{~Gb} / \mathrm{s}$ channel rates. The key to this breakthrough is the possibility to mitigate the most important linear transmission impairments, such as fiber link dispersion and polarization-mode dispersion. In coherent fiber-optic communication systems, the received optical signal is digitized through high-speed analog-to-digital converters and then processed using DSP algorithms. The input signal is recovered with the accuracy allowed by the channel noise and the transmission effects that are not equalized by DSP. After the mitigation of linear effects, noise and nonlinear impairments become the key factors in limiting the performance of coherent fiber-optic communication systems. In this Letter, through numerical modeling we illustrate for the optical communication community the possibility of using the inverse scattering transform (IST) (see [ $\underline{5}-\underline{9}]$ and references therein)-a technique developed few decades ago in other areas of physics-for mitigation of nonlinear impairments in coherent optical communications. Without loss of generality, we illustrate the application of the IST to transmission of a quadrature phase shift keying (QPSK) signal in a normal dispersion fiber link.

Note that, in general, the power of a signal transmitted through an optical fiber link is degraded by loss and has to be periodically recovered through optical amplification. In many important practical situations, averaging of such periodic loss and gain results in an effectively lossless propagation model-the nonlinear Schrödinger (NLS) equation [6,10]. Moreover, it has recently been experimentally demonstrated that fiber loss can be compensated continuously along a fiber span leading to effectively quasi-lossless transmission [11-13]. Therefore, the NLS equation is an important principal model that is used for demonstrating key techniques and approaches in optical fiber communications. In dimensionless units, the master equation reads $[\underline{6}, \underline{10}]$

$$
i U_{z}-\frac{s}{2} U_{t t}+|U|^{2} U=0
$$

where $s=\operatorname{sign}\left(\beta_{2}\right)= \pm 1$ for normal and anomalous dispersion, respectively $\left(\beta_{2}\right.$ is the group velocity dispersion coefficient); here we consider normal dispersion without loss of generality. The propagation distance $z=Z / L_{D}$ is normalized by the dispersion length $L_{D}=T_{s}^{2} /\left|\beta_{2}\right| ;$ time $t=T / T_{s}$-by the symbol rate $T_{s}$ and power-by the parameter $P_{0}$ chosen as $P_{0}=\left|\beta_{2}\right| /\left(\gamma T_{s}^{2}\right)$. We use the typical nonlinear coefficient $\gamma=1.3 \mathrm{~W}^{-1} \mathrm{~km}^{-1}$. The propagation distance in real-world units is $L[\mathrm{~km}]=z \times T_{s}^{2}\left[\mathrm{ps}^{2}\right] /\left|\beta_{2}\right|\left[\mathrm{ps}^{2} / \mathrm{km}\right]$, for instance, for $\beta_{2}=$ $5 \mathrm{ps}^{2} / \mathrm{km}$ and $T_{s}=25 \mathrm{ps}, L[\mathrm{~km}]=z \times 125[\mathrm{~km}]$. We focus here only on nonlinear signal distortion and will not include noise in this proof-of-principle illustrative analysis. An input optical signal $A(Z=0, T)=\sqrt{P_{0}} U(z=$ $\left.Z / L_{D}, t=T / T_{s}\right)$ at the beginning of the link $z=0$ is a QPSK modulated information pattern built from $N=$ 100 pulses: $U(z=0, t)=\sum_{n=1}^{N} c_{n} f_{0}\left(t-n T_{s}\right)$. Here, an input carrier pulse shape $f_{0}(t)$ could be any function. Without loss of generality, we consider a Gaussian pulse shape (though any pulse shape can be considered in a similar manner) $f_{0}(t)=\exp \left[-t^{2} /\left(2 \tau_{0}^{2}\right)\right] \times \exp [i \phi]$, where parameter $\tau_{0}$ is related to the pulse full width at half-maximum through $T_{\mathrm{FWHM}}=1.655 \tau_{0}$, and the phases of $c_{n}$ are generated randomly using QPSK modulation. Namely, $c_{n} /\left|c_{n}\right|$ are random numbers from the set $\{1, i,-1,-i\}$ and we consider $\left|c_{k}\right|=0.5$, which gives observable nonlinear effects. The average signal power in real-world units is $P_{\text {ave }}=\sqrt{\pi} P_{0}\left\langle\left|c_{k}\right|^{2}\right\rangle \tau_{0} / T_{s}$. The real and imaginary parts of the typical generated pattern are shown in Fig. 1.

The optical signal broadens after propagation through the fiber line. The absolute values of the amplitudes (square root of the signal power) of the original and the propagated signals are shown in Fig. 2. The inset demonstrates a constellation diagram of the input signal.

We use the Zakharov-Shabat spectral problem [5] (also known in other areas as the coupled mode theory equations) (2) for calculating the nonlinear reflection spectrum $r(\delta)$ [for a known $A(t)$ ]. The inverse problem is to determine signal $A(t)$ for a known $r(\delta)$ : 


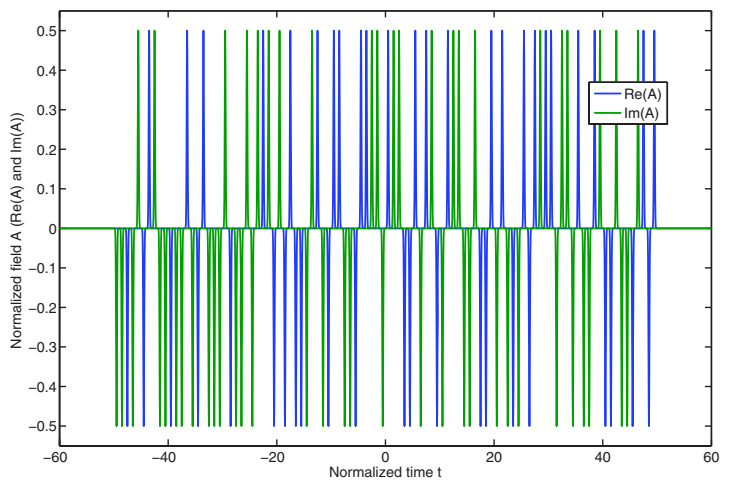

Fig. 1. Real (blue) and imaginary (green) parts of the input optical signal.

$$
\frac{d u}{d t}=+i \delta u+U(t) v, \frac{d v}{d t}=-i \delta v+U^{*}(t) u
$$

Here $u(t, \delta)$ and $v(t, \delta)$ are forward and backward propagating fields, respectively, and $\delta$ is a spectral parameter of the nonlinear Fourier transform (FT), playing the same role as frequency $\omega$ in the linear FT. The reflection spectrum $r(\delta)=v(0, \delta)$ is our focus of interest in solving the direct scattering problem.

For the purpose of comparison with the linear approach, we also compute the corresponding FT of the signals: $F U(\omega)$. After solving Eq. (2) for the input and the transmitted signal (Fig. 2), one can see (this is, certainly, the result of the inverse scattering theory) the following relation between $r(\delta)$ at $z=0$ and $L=50$ :

$$
r(\delta)_{z=50}=r(\delta)_{z=0} \times e^{2 i \times L \times \delta^{2}} .
$$

Note that the factor $L \times \delta^{2}$ (here in nondimensional units) is similar to the standard trivial evolution with distance of linear Fourier components for Eq. (1) $\propto z \times \omega^{2}$.

Figure $\underline{3}$ compares the spectra for both the nonlinear spectrum $\bar{r}(\delta)$ and the conventional linear FT, as well as the spectral phases-imaginary part of the expressions $\ln \left(r(\delta)_{z=50} / r(\delta)_{z=0}\right)$ and $\ln \left(F U(\omega)_{z=50} / F U(\omega)_{z=0}\right)$. From here we can reconstruct the original $r(\delta)_{z=0}$ and

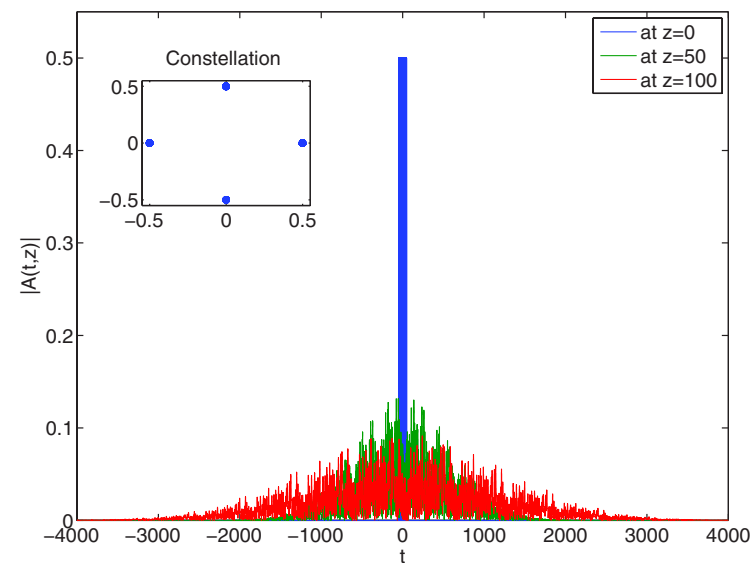

Fig. 2. Input (blue line) and propagated (green line at $z=50$, red line at $z=100$ ) optical signals; inset, constellation diagram (for $c_{n}$ ) of the input signal.
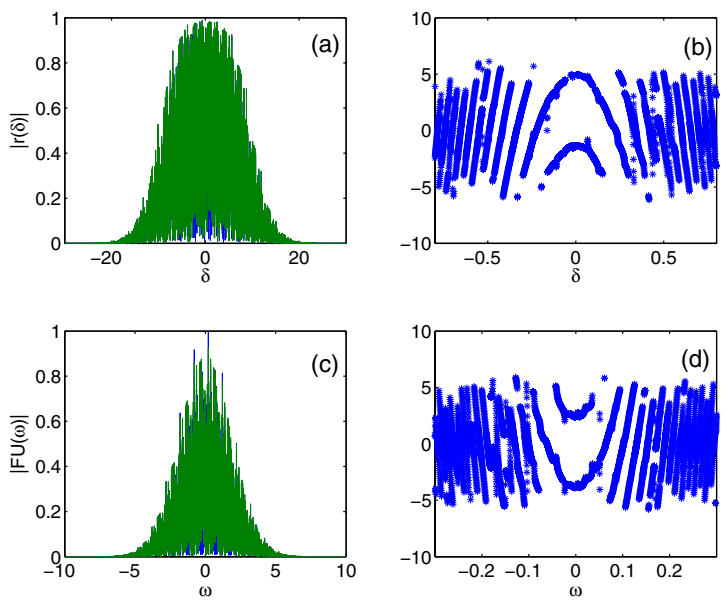

Fig. 3. (a) $\left|r(\delta)_{z=0}\right|$ and $\left|r(\delta)_{z=50}\right|$, (b) imaginary part of $\ln \left(r(\delta)_{z=50} / r(\delta)_{z=0}\right)$, (c) $\left|F U(\omega)_{z=0}\right|$ and $\left|F U(\omega)_{z=50}\right|$, and (d) imaginary part of $\ln \left(F U(\omega)_{z=50} /\left(F U(\omega)_{z=0}\right)\right.$.

$F U(\omega)_{z=0}$ by applying the corresponding inverse phase shifts [according to Eq. (3)].

Using the IST (as described in [14]) to solve Eq. (2) for a known $r(\delta)$, we reconstruct the original $A(z=0, t)$. At the same time, for comparison, we apply the linear inverse FT to the distorted field $F U(\omega)_{z=50}$.

When the nonlinear coefficient is small (very low signal powers), the reconstructed signals are identical, and the reconstruction via FT is very quick and efficient (numerical simulations for solving IST are several orders longer than with the inverse FT). However, with an increase of nonlinearity, the FT approach becomes less efficient, whereas the IST changes very little. Figure $\underline{4}$ shows eye diagrams of the reconstructed signals for different distances using both approaches. We can see that the IST approach [Figs. $4(\mathrm{e})-4(\mathrm{~h})$ ] provides better results than the spectral analysis via FT [Figs. 4(a)-4(d)]. With an increase of the propagation distance, the "eye" for Figs. 4(a)-4(d) starts "closing," while for the IST approach, the "eye" remains open. Figure 5 shows the corresponding constellation diagrams for those reconstructed signals.

We would like to discuss and elaborate on some important aspects of the key idea presented in this Letter. First, we would like to stress that the comparison is made only with the linear Fourier postprocessing, because
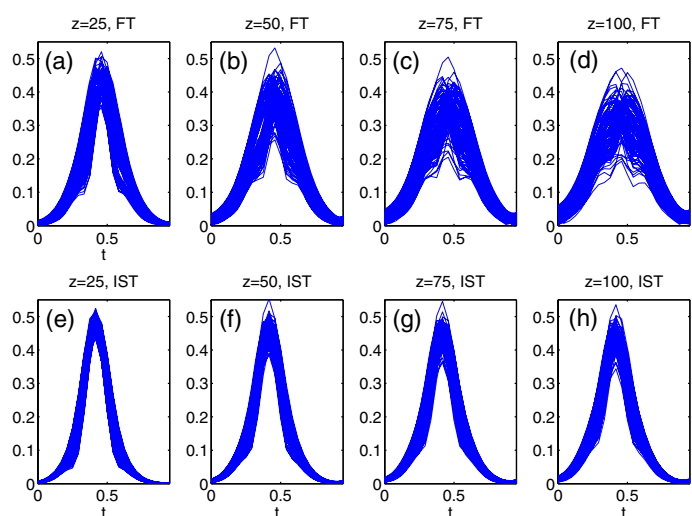

Fig. 4. Eye diagram for the signals reconstructed via FT, (a) at $z=25$, (b) at $z=50$, (c) at $z=75$, and (d) at $z=100$; and via IST, (e) at $z=25$, (f) at $z=50$, (g) at $z=75$, and (h) at $z=100$. 

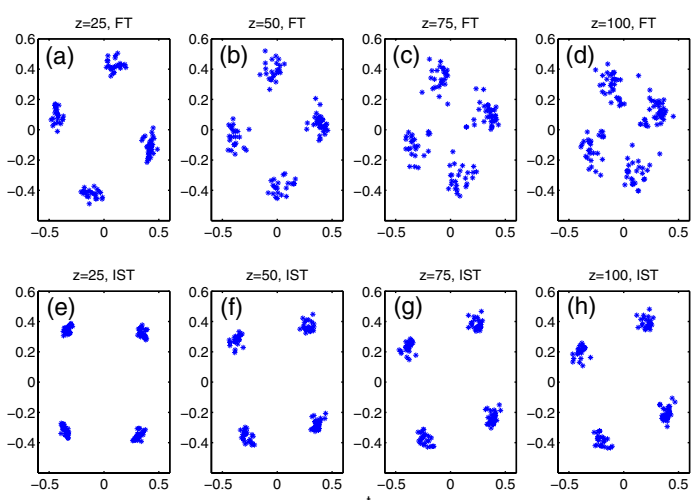

Fig. 5. Constellation diagram for the signals reconstructed via $\mathrm{FT}$, (a) at $z=25$, (b) at $z=50$, (c) at $z=75$, and (d) at $z=100$; and via IST, (e) at $z=25$, (f) at $z=50$, (g) at $z=75$, and (h) at $z=100$.

we discuss an approach that may potentially work in a similar manner, but without signal power restriction. The current trend in the field of optical telecommunications is using the dispersion noncompensated transmission regime, in which dispersion dominates over nonlinearity. The main linear time-invariant channel impairments such as chromatic dispersion and polarizationmode dispersion can be compensated by the adaptive linear equalization technique [2-4,15]. Though nonlinear transmission impairments due to fiber Kerr nonlinearity can be compensated by nonlinear backward propagation, this requires substantial computational efforts to model reverse signal channel propagation. The numerical challenges with nonlinear backward propagation are discussed, for instance, in [15-18]. The key technical difference between compensation of linear channel dispersion and nonlinear effects is that the linear FT compensates for accumulated channel dispersion analytically, without using any computer time for reverse propagation. The nonlinear FT (IST) illustrated in this Letter allows us to do the same with the nonlinear impairments. Using the nonlinear FT, evolution of the signal in $z$ can be accounted for analytically, even in the presence of nonlinearity. Of course, there is a price to pay for such an advantage-one has to solve the direct and the inverse scattering problem, instead of direct and inverse linear FTs, as in the linear channel equalization. Once more, in this Letter we aim to illustrate to the optical communication community the potential of this method that despite many challenging technical issues has the clear advantage of simultaneous compensation of both dispersion and nonlinearity.

We stress that we do not aim here to make a full comparison with the split-step-based nonlinear backward propagation method $[19,20]$. This is because the split-step method and the IST are based on conceptually different approaches. It is clear that $N \times \log N(N$ is the number of points) operations required for one elementary step in the split-step method are multiplied by the number of steps in $z$ that might be significant in long links. In the method discussed here, the IST, the propagation of the spectral data in $z$ can be accounted for analytically. Therefore, even if the number of operations in the time domain for solving direct and inverse scattering problems is larger than in split-step, the overall efficiency can be better. However, a fair comparison requires massive modeling and is beyond the scope and goal of this Letter.

In conclusion, we have illustrated through numerical modeling the recovery of a nonlinearly distorted signal using IST-based signal processing. In the IST technique a propagation part is trivial and technical problems are moved to the receiver and transmitter, offering the possibility of developing new approaches to DSP to mitigate nonlinear transmission impairments.

The support under the UK EPSRC Programme grant UNLOC EP/J017582/1, the grant of the Russian Ministry of Education and Science Federation N11.519.11.4001, and the Marie Curie IRSES program is gratefully acknowledged.

\section{References}

1. P. J. Winzer and R.-J. Essiambre, Proc. IEEE 94, 952 (2006).

2. S. J. Savory, Opt. Express 16, 804 (2008).

3. K. Kikuchi, IEICE Electron. Exp. 8, 1642 (2011).

4. D. S. Millar, S. Makovejs, C. Behrens, S. Hellerbrand, R. I. Killey, P. Bayvel, and S. J. Savory, IEEE J. Sel. Top. Quantum Electron. 16, 1217 (2010).

5. V. E. Zakharov and A. B. Shabat, Sov. Phys. JETP 34, 62 (1972).

6. A. Hasegawa and Y. Kodama, Solitons in Optical Communications (Oxford University, 1995).

7. S. V. Manakov, S. P. Novikov, L. P. Pitaevskii, and V. E. Zakharov, Theory of Solitons (Consultants Bureau, 1984).

8. M. J. Ablowitz and H. Segur, Solitons and the Inverse Scattering Transform (SIAM, 1981).

9. A. Hasegawa and T. Nyu, J. Lightwave Technol. 11, 395 (1993).

10. E. Iannone, F. Matera, A. Mecozzi, and M. Settembre, Nonlinear Optical Communication Networks (Wiley, 1998).

11. J. D. Ania-Castanon, T. J. Ellingham, R. Ibbotson, X. Chen, L. Zhang, and S. K. Turitsyn, Phys. Rev. Lett. 96, 023902 (2006).

12. T. J. Ellingham, J. D. Ania-Castanon, R. Ibbotson, X. Chen, L. Zhang, and S. K. Turitsyn, IEEE Photon. Technol. Lett. 18, 268 (2006).

13. J. D. Ania-Castanon, V. Karalekas, P. Harper, and S. K. Turitsyn, Phys. Rev. Lett. 101, 123903 (2008).

14. J. Skaar and O. H. Waagaard, IEEE J. Quantum Electron. 39, 1238 (2003).

15. E. M. Ip and J. M. Kahn, J. Lightwave Technol. 28, 502 (2010).

16. L. B. Du and A. J. Lowery, Opt. Express 18, 17075 (2010).

17. D. Rafique, M. Mussolin, M. Forzati, J. Mrtensson, M. N. Chugtai, and A. D. Ellis, Opt. Express 19, 9453 (2011).

18. L. Zhu and G. Li, Opt. Express 20, 14362 (2012).

19. O. V. Sinkin, R. Holzlohner, J. Zweck, and C. R. Menyuk, J. Lightwave Technol. 21, 61 (2003).

20. A. O. Korotkevich and P. M. Lushnikov, Opt. Lett. 36, 1851 (2011). 